\title{
Agôn
}

Revue des arts de la scène

5 | 2012

L'entrée en scène

\section{L'entrée en scène de la monstruosité dans Elephant Man de David Lynch}

\section{Marylin Marignan}

\section{(2) OpenEdition}

Journals

\section{Édition électronique}

URL : http://journals.openedition.org/agon/2343

DOI : 10.4000 /agon.2343

ISSN : 1961-8581

\section{Éditeur}

Association Agôn

\section{Référence électronique}

Marylin Marignan, «L'entrée en scène de la monstruosité dans Elephant Man de David Lynch », Agôn [En ligne], 5 | 2012, mis en ligne le 14 janvier 2013, consulté le 19 avril 2019. URL : http:// journals.openedition.org/agon/2343 ; DOI : 10.4000/agon.2343

Ce document a été généré automatiquement le 19 avril 2019

Association Agôn et les auteurs des articles 


\title{
L'entrée en scène de la monstruosité dans Elephant Man de David Lynch
}

\author{
Marylin Marignan
}

1 Inspiré de faits réels, le film de David Lynch Elephant Man, sorti en 1981, retrace les dernières années de la vie de John Merrick alias Elephant Man (John Hurt). Dans le Londres victorien, en 1884, un chirurgien, John Treves (Anthony Hopkins) découvre, dans une baraque foraine, un homme éléphant. Ce dernier affligé de multiples difformités est exhibé aux yeux de tous, contre quelques pièces de monnaies. Treves souhaite présenter ce spécimen à une assemblée de savants dont il fait partie.

Dans le long-métrage, la figure de John Merrick est centrale. Pourtant, le cinéaste décide de ne pas montrer d'emblée au spectateur le visage de l'homme éléphant. Il lui faut attendre plus d'une demi-heure après le début du film, pour qu'il soit réellement confronté à son aspect et à son regard. Malgré tout, dans cette première demi-heure, John Merrick va entrer en scène à différentes reprises et de différentes manières par un jeu de dissimulation retardant son apparition. En effet, nous ne verrons jamais distinctement le visage de l'homme éléphant, nous ne pourrons apercevoir que certaines parties de son corps.

3 Il s'agira donc ici de voir comment, par les différentes entrées en scène de l'homme éléphant, dans l'espace et dans le temps, David Lynch amène le spectateur à penser son regard sur la monstruosité.

\section{Penser le monstre}

4 Après le générique, Elephant Man débute par une série d'images et de sons oniriques. Cette séquence inaugurale, de par son montage très saccadé et ses surimpressions, marque une rupture $^{1}$. Elle évoque la vie prénatale et la naissance de la «créature » mais surtout, elle constitue, dans l'imaginaire de John, l'explication de sa monstruosité. Ainsi, dès les premières minutes du film, le cinéaste amène le spectateur à s'interroger sur le mystère qui entoure l'apparence de John. Cette séquence s'ouvre, par un gros plan, sur des yeux, annonçant la place centrale du regard dans l'appréhension du monstre. 
Elephant Man de David Lynch, 1981

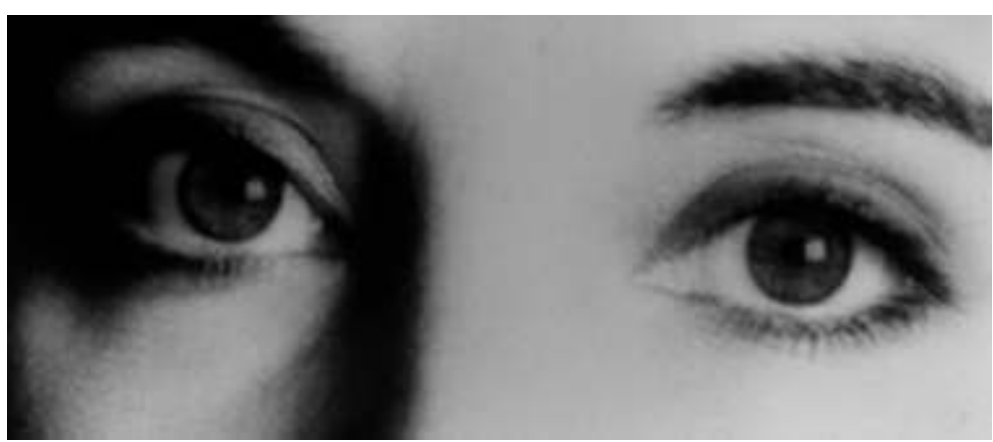

5 Par la suite, le portrait photographique d'une femme apparaît. La mélodie d'une boîte à musique accompagne ses images avant de s'arrêter brusquement. Sur le visage féminin, des sons angoissants, annonçant une suite dramatique, vont faire leur apparition. Des éléphants envahissent l'écran. La jeune femme va être violemment projetée à terre par un coup de trompe. Elle hurle en agitant sa tête de gauche à droite. On entend des barrissements qui pourraient provenir de sa bouche. Après un fondu au noir, sur des cris de bébé, un nuage de fumée apparaît alors comme la métaphore de la naissance de John. Dans son ouvrage Mythes, Monstres et Cinéma: aux confins de l'humanité (2008) ${ }^{2}$, Olivier Rachid Grim voit deux lectures possibles de cette séquence. Premièrement, la difformité de John ne serait pas liée à une maladie mais serait la conséquence d'un malheureux accident : l'attaque puis le piétinement de sa mère par les éléphants. Histoire incroyable mais néanmoins possible : le fotus aurait été abîmé d'où les difformités de John. Ce récit donne donc une justification à son état. Le second angle de lecture serait de l'ordre du fantastique et s'inscrirait dans une définition mythologique du monstre : un être hybride. La femme aurait été attaquée puis violée par le pachyderme. Suite à cette union sexuelle, John serait né ${ }^{3}$. La signification du mot "monstre " a évolué au cours du temps. De l'antiquité jusqu'à la fin du XVII ${ }^{\mathrm{e}}$ siècle, ce terme renvoyait au fabuleux ${ }^{4}$. La notion d'hybridité fait référence, dans l'imaginaire collectif, à diverses créatures terrifiantes appartenant à la mythologie: le Minotaure (corps d'homme/tête de taureau), les Gorgones (chevelures de serpents), les Centaures (corps de chevaux/tête d'homme), les Sphinx (corps de lions/buste et tête de femmes)... Dans l'esprit du spectateur, un lien se crée automatiquement entre ces êtres et John. Ainsi, dès les premiers plans du film, David Lynch guide notre pensée en présentant John Merrick comme un monstre effroyable pouvant rivaliser avec des êtres mythologiques. Cette séquence permet au cinéaste de nous préparer à la vision du monstre, sans pour autant nous le dévoiler. En retardant son entrée en scène, il nous incite à construire l'image fantasmée d'une créature entre humanité et animalité.

Dans la première partie du film, John Merrick est exhibé comme phénomène de foire par son propriétaire Bytes. Contre de l'argent, ce dernier invite les curieux à venir contempler une « merveille de la nature ${ }^{5}$ » : un homme éléphant. Entre le XIX et le milieu $\mathrm{du} \mathrm{XX}^{\mathrm{e}}$ siècle, les exhibitions de monstres, également appelées "side-shows ", sont très en vogues et très appréciées en Amérique et en Europe ${ }^{6}$. Dans le film, sur l'enseigne de la baraque foraine abritant les phénomènes et l'homme éléphant, le mot «Freaks » (« monstres ») est noté en lettres majuscules. Un gros plan insiste sur cette dénomination. Le cinéaste s'appuie donc d'emblée sur l'imaginaire collectif en associant la monstruosité à John Merrick. Le docteur John Treves espère pouvoir observer ce spécimen. Malgré une 
pancarte indiquant "no entry» (« ne pas entrer»), il s'engouffre sous le chapiteau à la recherche de cette "bête» énigmatique. Au détour de longs corridors, d'autres phénomènes de foire sont visibles : une femme à barbe, un homme vent, un fœtus dans un bocal... Ces différentes exhibitions attirent des foules ne pouvant contenir leurs rires. Bien qu'impressionnantes, ces représentations ne retiennent pourtant pas l'attention du médecin et ne semblent pas à la hauteur de l'attraction attendue: le seul véritable spectacle étant la rencontre avec l'homme éléphant. Dans ce passage, la monstruosité est définie comme un "raté» de la nature. Au siècle des Lumières, les monstres n'appartiennent plus au domaine du fabuleux mais constituent un écart par rapport à la norme. Au début du XIX siècle, un basculement s'effectue : les monstres sont davantage assimilés à une erreur de la nature ${ }^{7}$. Notre tentative de voir John Merrick, ainsi que celle de Treves, échouent. En effet, des policiers font fermer ce commerce. Cette exhibition est la seule à être interdite pour cause "d'atteinte à la décence ». Les autorités s'insurgent contre cette mise en scène qu'elles pensent trop dégradante pour la foule, pour l'homme éléphant et par conséquent, pour le spectateur du film. Les excroissances de la créature outrepassant les limites du convenable: indice supplémentaire d'une monstruosité indéfinissable. L'insistance sur les visages des personnes sortant choquées, en pleurs de cette exposition, juste avant qu'elle ne soit fermée, renforce ce sentiment d'ignominie. La première entrée en scène du phénomène est donc avortée. Elle marque une étape supplémentaire dans notre approche de ce corps: nous ne sommes plus en mesure de l'appréhender. Alors que la séquence inaugurale éveillait notre curiosité et notre imagination, dans ce passage, David Lynch s'emploie à déconstruire notre perception de cette créature. L'intervention d'une autorité politique dans l'interruption de l'attraction signifie que les difformités de cet être sont d'une telle infamie que nous ne sommes pas en mesure de les saisir : le physique de l'homme éléphant est plus atroce que ce que nous avions pu penser. Ainsi, notre volonté de voir cet individu parce qu'il est insaisissable, se fait plus forte, « le visuel [...] [étant] ce par quoi la vérification de l'existence de la chose peut se faire ${ }^{8} »$.

\section{Désir de voir le monstre}

7 John Treves, n'ayant pas assouvi son désir de voir cet être anormal, le fait rechercher. Une fois John Merrick retrouvé, le docteur paye une grosse somme d'argent à son montreur Bytes, pour avoir une séance privée. Cette dernière a lieu dans la pénombre d'une cave. Tous les artifices d'une représentation théâtrale sont réunis. L'homme éléphant est caché derrière un rideau. Une faible lumière éclaire le devant de la scène improvisée. Bytes commence le spectacle par une brève présentation de sa créature. Filmé en plan fixe, il raconte l'histoire de John Merrick. Cette démarche permet de préparer Treves mais également le spectateur du film (qu'il interpelle par un «mesdames, messieurs ») à la terrible vision du monstre. Ce dernier se retrouve alors dans la même position que Treves: il a payé sa place de cinéma pour voir l'effroyable spectacle de l'homme éléphant. Le public désire voir à quoi ressemble John Merrick : il est venu voir le film, en grande partie, dans ce but. Les producteurs avaient préparé la sortie de cette œuvre en misant justement sur la curiosité des masses. Ainsi, ni sur les affiches du film, ni dans les différentes bandes annonces, le visage de l'homme éléphant n'était visible. Sur l'affiche principale du film, au centre, un homme apparaissait dissimulé sous une cape, une cagoule et une casquette. Les différents supports indiquaient seulement 
que le film était basé sur une histoire vraie et que John Merrick était un homme doté d'intelligence. Par ce procédé s'amorce une pulsion scopique «impliquant le besoin de voir et le désir de regarder ${ }^{9}$ ». Cette pulsion sera prolongée et entretenue par des jeux de dissimulations et de regards entre John, le spectateur du film et les personnages de la diégèse qui seront développés notamment lors des différentes tentatives d'entrées en scène de John Merrick. Entre attraction, attirance et répulsion, le monstre exerce une fascination: tout comme Treves, nous avons besoin d'assouvir notre désir d'être confrontés à l'horrible créature, un réel désir qui ne va pourtant pas aboutir. En effet, l'entrée en scène de John ne va pas nous donner satisfaction. À la fin de la présentation de Bytes, le rideau s'ouvre. C'est la première fois que John apparaît à l'écran depuis le début $\mathrm{du}$ film. Et après une attente de quinze minutes, nous avons seulement droit à une vision rapide de son corps plongé dans l'obscurité. Il y a donc déception pour le spectateur qui ne peut trouver que des indices de l'apparence de John dans le regard de Treves. Le médecin apparaît bouleversé par la vision de l'homme éléphant. Une horreur muette se lit sur son visage, la caméra se focalisant sur ses yeux. Cette scène se compose d'un travelling avant, avec un gros plan sur son visage. Presque inconsciemment, une larme coule sur sa joue. Ce passage s'achève par un fondu au noir. Son rythme lent permet d'observer et de s'imprégner, au travers de l'expression de Treves, de l'émotion découlant de la vision de la bête. La musique, présente uniquement dans la dernière partie, vient renforcer l'aspect dramatique de cette apparition. Le spectateur cherche des réponses sur l'apparence de John en analysant les réactions sur le visage du médecin. Treves devient alors un pôle d'identification ${ }^{10}$. Nous nous approprions son attitude et son émotion. Michel Chion dans son ouvrage consacré à David Lynch parle de : " plans de déconvenue insistant sur la déconfiture d'un personnage par une image appuyée de sa réaction, qui le désigne comme cible du public $»^{11}$ Dans ce passage, Bytes est l'instigateur de l'entrée en scène de John Merrick. Ce dernier ne semble pas désirer un face à face avec le spectateur : il suit les ordres de son propriétaire qui lui ordonne de se montrer. Cette première entrée en scène appelle la suivante car notre désir de voir n'a pas été satisfait. Cette apparition furtive du monstre dans la pénombre permet au cinéaste de réintroduire la notion d'imaginaire dont il s'était éloigné afin d'insister sur la nature indicible du spectacle. Dans un premier temps, en dévoilant des parties du corps de cet être (même si nous avons du mal à les cerner), David Lynch réactive notre besoin de nous approprier l'image de cette créature. Puis, dans un second temps, l'appréhension de ce corps se fait par l'identification à Treves auquel nous nous raccrochons pour concevoir cet être différent. Le travail sur le rythme de la séquence est primordial : juxtaposition de plans rapides sur le corps de John Merrick, permettant de relancer notre besoin de voir et des plans plus longs, insistants sur la réaction du médecin, pour penser les difformités de la créature. 
Elephant Man de David Lynch, 1981

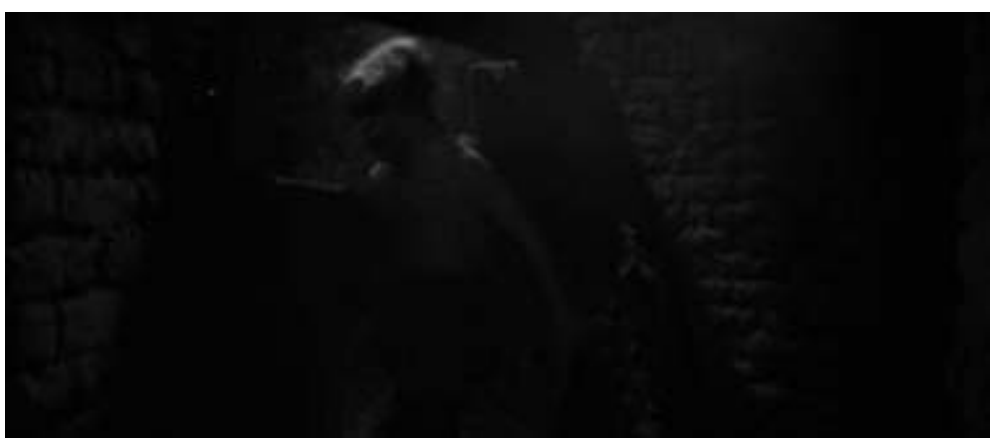

Elephant Man de David Lynch, 1981

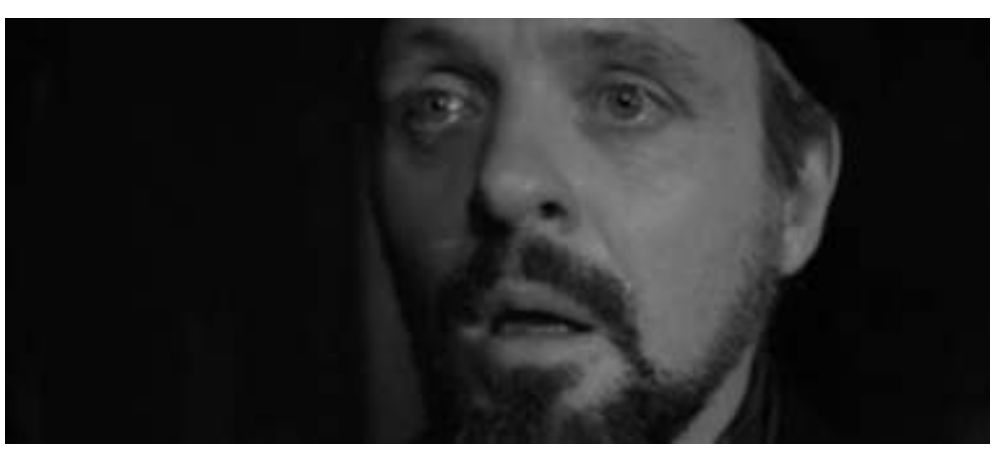

8 La seconde rencontre avec John Merrick aura lieu deux minutes plus tard. Treves a payé pour le voir en consultation. La créature va donc se rendre à l'hôpital. Au début du XIX siècle, les monstres intéressent les scientifiques, ils deviennent alors des «objets de science à part entière, en ce sens qu'ils vont être nommés et classés à partir de données anatomiques ${ }^{12}$ Durant cette séquence, le corps de John est dissimulé sous une cape, une cagoule et une casquette difforme. Ces quelques artifices séparent le spectateur de l'horreur. La vision d'une main d'apparence normale, tenant la canne qui soutient l'individu, questionne et intrigue. Avant de le voir, nous l'entendons. Sa respiration semble très difficile. Il émet des sortes de râles. Dans un premier temps, le cinéaste nous contraint donc à imaginer le corps de John par le son puis, nous permet enfin de voir cette masse cachée sous des haillons, qui peine à se déplacer, malgré l'aide d'une canne. L'allusion d'un petit garçon, à l'odeur nauséabonde que dégage John, participe à construire une image négative et répulsive de cet être. Michel Chion voit un parallèle entre «la machinerie corporelle usée et souffrante et les évocations industrielles du film $»^{13}$ Le choix de David Lynch de tourner un film en noir et blanc et en format cinémascope s'insère dans cette volonté de décrire une civilisation industrielle en plein essor et permet d'aborder la créature avec plus de distance et de dignité ${ }^{14}$. Dans cette séquence, John se retrouve une fois de plus mis en scène. Il pénètre dans l'hôpital par une porte située à gauche de l'écran. Le spectateur ne le voit pas la franchir, il entend simplement son ouverture. Le cocher, accompagnant la créature, l'invite à s'avancer dans le hall d'accueil de l'hôpital. À son entrée, les discussions des malades et du personnel soignant s'arrêtent. Le spectacle a commencé. Tous les regards se tournent vers le mystérieux personnage. Les patients, assis sur des bancs disposés les uns derrière les autres, scrutent la difficile avancée de John. Ils font face à une représentation théâtrale. Les coulisses de la scène sont évoquées par l'entrée et la sortie de John. Le dernier plan 
filmé en forte plongée rappelle les galeries présentes dans une salle de théâtre. La marche très lente de John, en direction de l'infirmière, laisse tout le temps nécessaire aux patients de l'hôpital, ainsi qu'au spectateur du film, d'imaginer ce qui se cache sous ces vêtements. Dans ce passage, c'est la différence plus que le monstrueux qui est évoquée. Les patients n'ont pas conscience d'être face à l'homme éléphant : la différence comme le monstrueux intriguent, attirent les regards et isolent les individus. La fin de la séquence se déroule dans le cabinet du docteur Treves. Après une série de questions auxquelles John Merrick semble incapable de répondre, le médecin s'approche de lui pour enlever sa cagoule. Alors que nous sommes sur le point de découvrir le visage de l'homme éléphant, la scène se termine par un fondu au noir. Nous ne sommes pas encore conviés à regarder l'étrange créature. Dans cette séquence, le cinéaste nous invite à nous concentrer sur la créature et non pas sur le regard des personnages de la diégèse (comme lors de la première entrée en scène). Cette deuxième vision, plus longue, de l'homme éléphant, nous laisse davantage de temps pour tenter de nous représenter son corps en nous fondant sur des indications qui nous sont données tout au long de cette scène. Pour autant, cette deuxième entrée en scène déçoit, une fois de plus, le spectateur dont la scopophilie définie comme la " perversion liée à l'exacerbation de la pulsion scopique ${ }^{15}$ ", n’a pas encore été assouvie.

Elephant Man de David Lynch, 1981

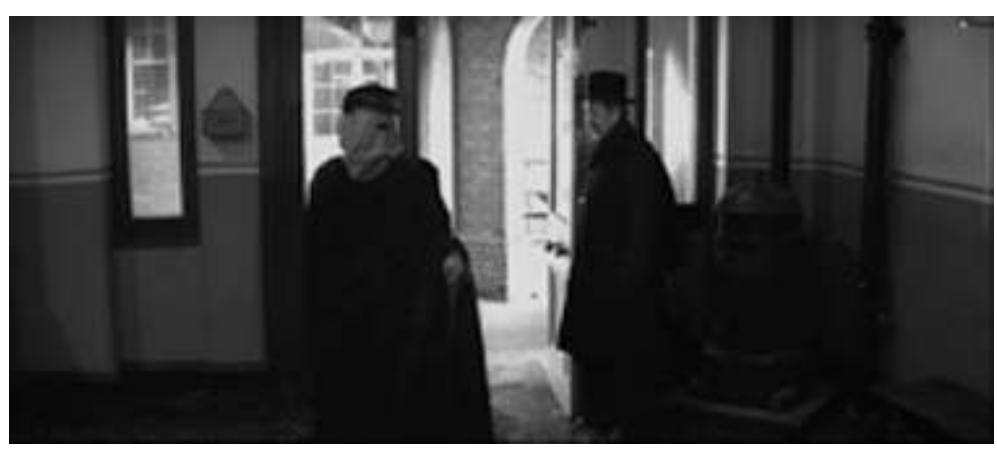

Elephant Man de David Lynch, 1981

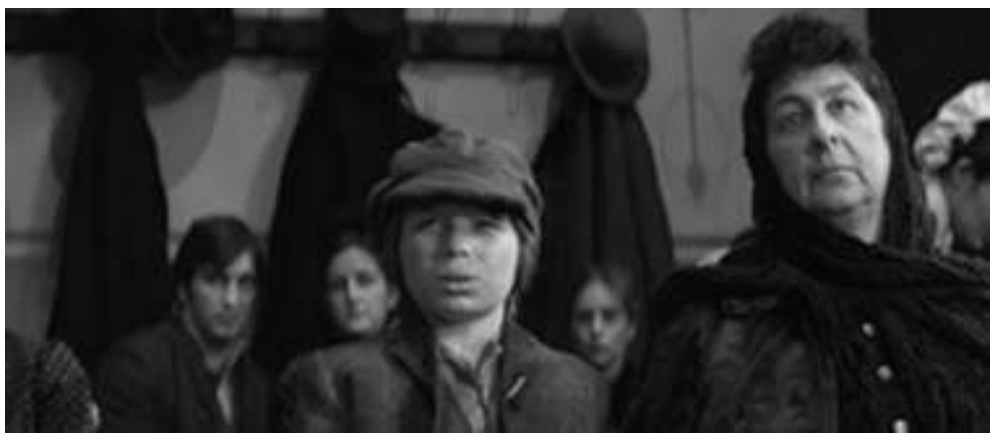

Le cinéaste dissimule le corps de John, d'une troisième façon. Lors d'une présentation de l'homme éléphant à une société de pathologie, sa silhouette déformée ne sera visible qu'à travers un paravent. Treves, fasciné par la créature, fait partager sa découverte à ses confrères. Cette conférence est prétexte à une nouvelle exhibition de l'homme éléphant avec faisceau lumineux dirigé en sa direction, lever de rideau, applaudissements... La silhouette qui apparaît en ombre chinoise fait penser à un spectacle de vues animées, l'ancêtre du cinématographe. Les scientifiques peuvent admirer le spécimen tout en 
écoutant la description très précise de Treves sur les différentes difformités dont est atteint John. Il y a une focalisation sur les regards. Le spectateur cherche des réponses à ses questions dans l'émotion qu'il peut lire sur le visage des médecins. Une fois de plus, le rythme de la séquence laisse le temps d'examiner avec soin les regards attentifs des chercheurs et la silhouette déformée de John. Le commentaire de Treves associé à la vision partielle du corps, permet d'avoir une idée plus précise de ces malformations. Malgré tout, le spectateur est frustré de ne pas pouvoir apprécier totalement le spectacle. Son désir de voir est d'autant plus aiguisé que certaines interpellations de Treves aux scientifiques peuvent lui être tout autant adressées : «Vous voyez bien?", "Vous êtes assez prêt ? »... Cette troisième entrée en scène est encore une fois contrainte pour que les scientifiques puissent voir et étudier ce corps monstrueux. Dans cette scène, paradoxalement, le spectateur sait exactement à quoi ressemble le corps de John sans l'avoir réellement vu. David Lynch entretient le désir de contempler la monstruosité en exacerbant notre curiosité par des accumulations descriptives sur les caractéristiques physiques de l'homme éléphant. Le cinéaste focalise notre regard sur le corps de John mais également sur les regards stupéfaits des médecins. De cette façon, cet extrait conjugue les éléments mis en place lors des deux premières entrées en scène du monstre.

Elephant Man de David Lynch, 1981

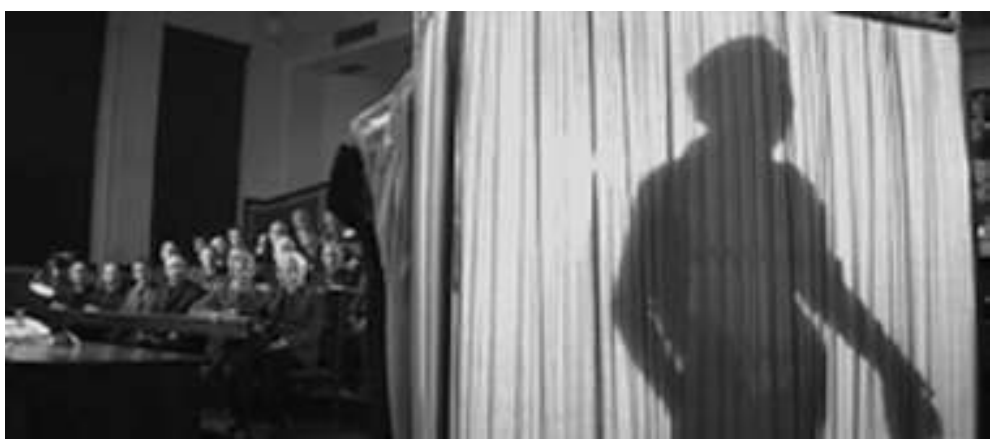

Elephant Man de David Lynch, 1981

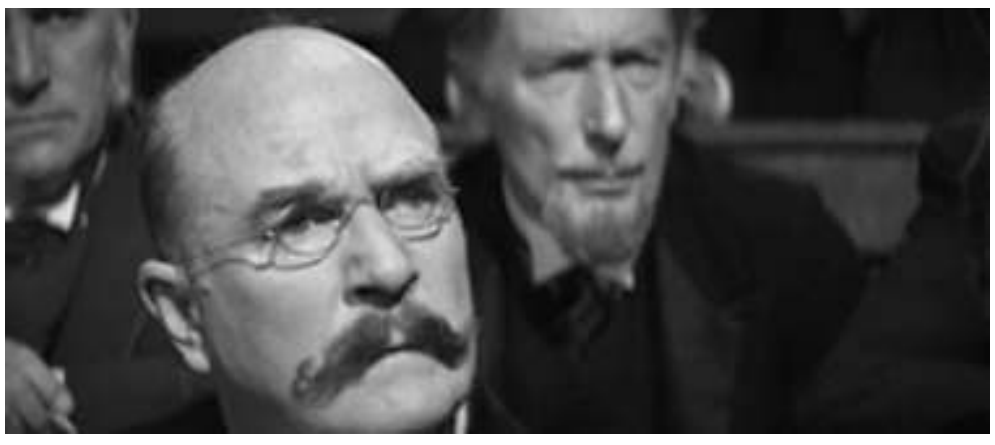

\section{Le mystère révélé : la peur du monstre}

Depuis le début du film, nous savons que le mystère de l'apparence de John finira par nous être révélé. Cette rencontre se fait une demi-heure après le début du film. Treves a installé John dans une petite chambre de l'hôpital à l'écart des autres patients. David Lynch convoque tous les procédés présents dans un film d'horreur traditionnel : la nuit, 
les couloirs déserts, une jeune fille seule, des sons inquiétants... Un montage alterné va montrer d'un côté une discussion entre Treves et le directeur de l'hôpital, et de l'autre, une jeune infirmière devant apporter une assiette de céréales à John. Avant que le monstre ne soit dévoilé, Treves demande au directeur : « Vous voulez le voir? », question qui s'adresse autant au directeur qu'au spectateur du film. Ce dernier sait qu'il va enfin pouvoir contempler son visage. La jeune fille monte lentement les escaliers menant à la chambre de John. Des sons angoissants accompagnent sa douloureuse ascension. Elle est tétanisée. Arrivée sur le seuil de la porte, elle hésite à entrer. L'émotion et la tension émanant de cette scène sont palpables. L'apparition se fait proche. Elle ouvre la porte. Un plan rapide montre John qui s'enfonce dans son lit et essaye de se cacher avec sa couverture. La jeune fille hurle et fait tomber l'assiette à terre. Retour sur John qui crie également par peur. Il y a renversement. Plutôt que l'horreur, le film d'épouvante suscite le désarroi : l'effrayant monstre qui devait nous faire peur est un être démuni lui-même terrorisé. «Le monstre a peur ", écrivait en 1981 Serge Daney dans un article éponyme consacré au film. Formule singulière mettant en évidence le renversement de perspective à l'œuvre dans cette séquence, tant il est généralement admis que les monstres provoquent la peur plus qu'ils ne la ressentent. Et l'essayiste de préciser, en commentant cette rencontre : « le spectateur le voit - vraiment - pour la première fois, mais ce qu'il voit aussi c'est que ce monstre censé lui faire peur a peur lui-même »" ${ }^{16}$. À ce moment du film s'opère donc un basculement : ce n'est plus notre peur et celle des personnages qui est au centre du film mais celle de John. Pour David Lynch, cette attente était primordiale pour donner le temps au spectateur de s'habituer à l'homme éléphant: "On ne voit vraiment John Merrick que lorsqu'on a eu le temps de s'attacher à lui. Il fallait arriver à dépasser les apparences, car c'est là le problème de fond, cette distorsion entre l'apparence et la réalité ${ }^{17} \mathrm{Il}$ y a une évolution quant au regard que pouvait avoir le spectateur sur John. Pour la première fois, il apparaît comme une personne ayant peur du regard qu'elle peut renvoyer aux autres. La révélation de son corps se fait par l'intermédiaire de la jeune infirmière qui ouvre la porte derrière laquelle il se réfugiait. John ne désire pas cette entrée en scène ni les autres. Cette séquence nous en donne la raison : il a peur du regard des autres, il a peur de faire peur. En retardant la véritable entrée en scène de l'homme éléphant, David Lynch nous laisse le temps nécessaire pour penser la monstruosité comme spectacle insoutenable et terrifiant d'un être différent. La révélation du corps de John Merrick déconstruit l'appréhension fantasmée du monstre. Nous prenons alors conscience que notre regard sur la monstruosité est erroné. Une redéfinition de cette notion est alors engagée.

Elephant Man de David Lynch, 1981

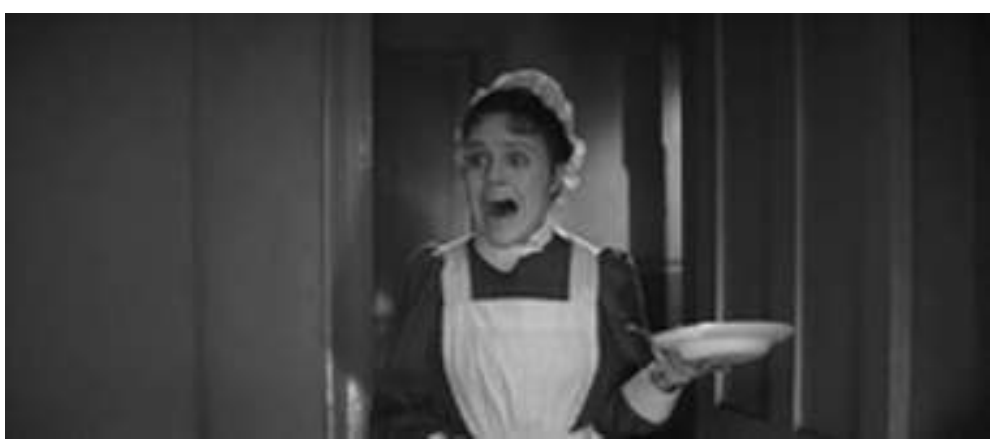


Elephant Man de David Lynch, 1981

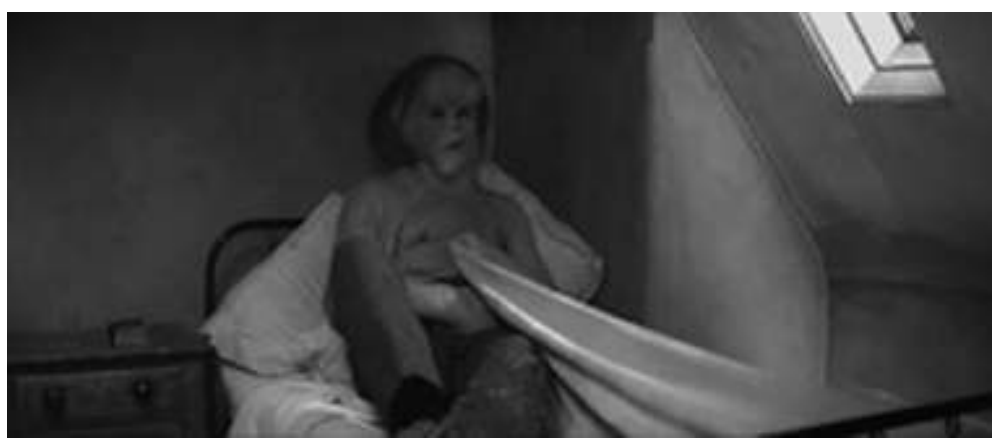

11 Ainsi, en traitant l'entrée en scène de façon peu conventionnelle, dans le temps et par étapes, David Lynch place la construction d'un regard sur la monstruosité comme l'un des enjeux centraux de sa mise en scène. Tour à tour créature mythologique, phénomène de foire, infirme ou objet scientifique, John Merrick apparaît finalement comme un être apeuré. Progressivement, le cinéaste guide et impose au spectateur que nous sommes plusieurs lectures du monstre nous amenant ainsi à repenser notre perception de la monstruosité.

\section{BIBLIOGRAPHIE}

AUMONT Jacques. L'Image [1990]. Paris : Armand Colin : 2005. Page 92. 254 pages.

CHION Michel. David Lynch [1992]. Nouvelle édition augmentée. Paris : éd. de l'Étoile/Cahiers du cinéma, 2001. (Coll. Auteurs). 288 pages.

DANEY Serge. « Le monstre a peur », in Les Cahiers du cinéma. N³22, avril 1981. Page 33.

DAVID Lynch. Entretien avec TRANCHANT Marie-Noëlle paru dans le Figaro, le 6 avril 1981.

DESBARATS Carole et DIDIER-WEILL Alain. Dossier « collège au cinéma » n75 : Elephant Man de David Lynch. Édité pour le compte du Centre National de la Cinématographie par les Films de l'Estran.

FISCHER Jean-Louis. Monstres : histoire du corps et de ses défauts. Paris : Syros Alternatives, 1991. 126 pages.

GRIM Olivier Rachid. Mythes, Monstres et Cinéma : aux confins de l'humanité. Grenoble : Presses universitaires de Grenoble, 2008. 311 pages.

PAJOT Stéphane. De la femme à barbe à l'homme-canon. Phénomènes de cirque et de baraque foraine. Le Château d'Olonne : éd. D’Orbestier, 2003. 195 pages.

PIERRON Agnès. Dictionnaire de la langue du cirque. Des mots dans la sciure. Paris : éd. Stock, 2003. 593 pages. 
RUDEFOUCAULD Alain Julien. « Le monstre et le visuel. Monstruosité du cinéma », In Imaginaire \& Inconscient. $\mathrm{N}^{\circ} 13$, janvier 2004. Page 109-115. URL : www.cairn.info/revue-imaginaire-etinconscient-2004-1-page-109.htm

Dossier « cinéma et pédagogie » : Elephant Man de David Lynch, novembre 1993.

Dossier pédagogique « Ciné Santé $95: 1^{\mathrm{er}}$ festival de cinéma et d'éducation pour la santé. » : Elephant Man de David Lynch. 1993.

\section{NOTES}

1. La séquence finale est également construite sur le même schéma. Elle répond à ce moment et offre une conclusion lyrique au film.

2. GRIM Olivier Rachid. Mythes, Monstres et Cinéma : aux confins de l'humanité. Grenoble: Presses universitaires de Grenoble, 2008, p. 211-212.

3. En réalité, John Merrick était atteint d'une maladie génétique héréditaire: la neurofibromatose. Cette dernière se manifeste par l'apparition de tumeurs disséminées sur tout le corps et parfois à l'intérieur de l'organisme. De nos jours, la chirurgie esthétique et la chimiothérapie permettent de réduire ces excroissances. Voir sur ce sujet : Dossier pédagogique «Ciné Santé $95: 1^{\text {er }}$ festival de cinéma et d'éducation pour la santé. »: Elephant Man de David Lynch. 1993.

4. FISCHER Jean-Louis. Monstres : histoire du corps et de ses défauts. Paris : Syros Alternatives, 1991, p. 8.

5. Le terme "merveilles de la nature ", inventé par Barnum, désigne les phénomènes exhibés dans les foires. PIERRON Agnès. Dictionnaire de la langue du cirque. Des mots dans la sciure. Paris : éd. Stock, 2003, p. 448.

6. PAJOT Stéphane. De la femme à barbe à l'homme-canon. Phénomènes de cirque et de baraque foraine. Le Château d'Olonne : éd. D’Orbestier, 2003, p. 10.

7. FISCHER Jean-Louis, op. cit., p. 8 à 10.

8. RUDEFOUCAULD Alain Julien. «Le monstre et le visuel. Monstruosité du cinéma», In Imaginaire \& Inconscient. $\mathrm{N}^{\circ} 13$, janvier 2004, pp. 109-115. URL : http://www.cairn.info/revueimaginaire-et-inconscient-2004-1-page-109.htm

9. AUMONT Jacques. L'Image [1990]. Paris : Armand Colin : 2005, p. 92.

10. CHION Michel. David Lynch [1992]. Nouvelle édition augmentée. Paris : éd. de l'Étoile/Cahiers du cinéma, 2001. (Coll. Auteurs), p. 73.

11. Idem, p. 74.

12. FISCHER Jean-Louis, op.cit. p. 8.

13. CHION Michel, op. cit., p. 68.

14. Entretien de David LYNCH avec TRANCHANT Marie-Noëlle paru dans le Figaro, le 6 avril 1981.

15. AUMONT Jacques, op. cit., p. 94.

16. DANEY Serge. «Le monstre a peur », in Les Cahiers du cinéma. $\mathrm{N}^{\circ} 322$, avril 1981, p. 33.

17. Entretien de David LYNCH avec TRANCHANT Marie-Noëlle, art. cité. 


\section{RÉSUMÉS}

À partir d'analyses précises de séquences, cet article se propose d'étudier les différentes entrées en scène de l'homme éléphant, dans le film de David Lynch Elephant Man. Dans cette œuvre, le cinéaste place monstruosité et regard spectatoriel comme pivots de la construction d'une mise en scène par étapes. Confrontant le spectateur à plusieurs définitions possibles du monstre (créatures mythologiques, objets scientifiques...), l'homme éléphant s'avère finalement être une personne démunie et apeurée, souffrant de l'image qu'elle renvoie aux autres. Ainsi, en traitant l'entrée en scène, dans le temps et dans l'espace, David Lynch amène le spectateur à repenser sa propre notion de monstruosité.

INDEX

Mots-clés : cinéma, Lynch (David)

\section{AUTEUR}

\section{MARYLIN MARIGNAN}

Marylin Marignan prépare un doctorat en études cinématographiques. Sa thèse porte sur l'évolution de la fréquentation des théâtres et des cinémas à Lyon (1929-1939). Parallèlement à son doctorat, elle est chargée de cours à l'Université Lumière Lyon 2 au sein du département des Arts de la Scène, de l'Image et de l'Écran. 